PROCEEDINGS OF THE

AMERICAN MATHEMATICAL SOCIETY

Volume 129, Number 8, Pages 2441-2443

S 0002-9939(01)05801-4

Article electronically published on January 23, 2001

\title{
BOREL COMPLEXITY OF THE SPACE OF PROBABILITY MEASURES
}

\author{
ABHIJIT DASGUPTA
}

(Communicated by Andreas R. Blass)

\begin{abstract}
Using a technique developed by Louveau and Saint Raymond, we find the complexity of the space of probability measures in the Borel hierarchy: if $X$ is any non-Polish Borel subspace of a Polish space, then $P(X)$, the space of probability Borel measures on $X$ with the weak topology, is always true $\mathbf{\Pi}_{\xi}^{\mathbf{0}}$, where $\xi$ is the least ordinal such that $X$ is $\boldsymbol{\Pi}_{\xi}^{0}$.
\end{abstract}

\section{INTRODUCTION}

For $X$ a separable metric space, let $P(X)$ be the space of probability Borel measures on $X$ with the usual topology of weak convergence, so that $P(X)$ is also a separable metrizable space. Many results relating the descriptive complexities of $X$ and $P(X)$ are classical and standard. For example (see [1], [5]), $X$ is compactmetrizable (resp. Polish) iff $P(X)$ is compact-metrizable (resp. Polish), $X$ is Borel (resp. projective) iff $P(X)$ is Borel (resp. projective), etc. Beyond the domain of Borel sets we have: $X$ is analytic (resp. co-analytic) iff $P(X)$ is analytic (resp. coanalytic), which follows from results of Kechris [2, and this also extends to every level of the projective hierarchy under additional set-theoretic hypotheses. Shreve's theorem ([6]) establishes the same equivalence for each level of the $C$-hierarchy of Selivanovski.

In this note we prove a similar result for the Borel hierarchy. It is known ([2]) that, for $\alpha \geq 2, P(X)$ is $\boldsymbol{\Pi}_{\boldsymbol{\alpha}}^{\mathbf{0}}$ iff $X$ is $\boldsymbol{\Pi}_{\boldsymbol{\alpha}}^{\mathbf{0}}$; hence if $X$ is $\boldsymbol{\Sigma}_{\boldsymbol{\alpha}}^{\mathbf{0}}$, then $P(X)$ is $\boldsymbol{\Pi}_{\boldsymbol{\alpha}+\mathbf{1}}^{\mathbf{0}}$. This suggests the question: Is this the best bound? More generally, for $\Gamma$ and $\Gamma^{\prime}$ any two intrinsic Borel pointclasses, we ask: If $X$ is in $\Gamma$, is $P(X)$ in $\Gamma^{\prime}$ ? It is also easy to find, for each $\alpha \geq 3$, an example of a $P(X)$ which is true $\boldsymbol{\Pi}_{\boldsymbol{\alpha}}^{\mathbf{0}}$ : just take $X$ to be any space which is true $\boldsymbol{\Pi}_{\boldsymbol{\alpha}}^{\mathbf{0}}$; since $X$ is always embedded in $P(X)$ as a closed subset, $P(X)$ cannot be $\boldsymbol{\Sigma}_{\boldsymbol{\alpha}}^{\mathbf{0}}$ lest $X$ be $\boldsymbol{\Sigma}_{\boldsymbol{\alpha}}^{\mathbf{0}}$. This suggests the question: For which $\alpha \geq 3$ can we find $X$ such that $P(X)$ is true $\boldsymbol{\Sigma}_{\boldsymbol{\alpha}}^{\mathbf{0}}$ (or true $\boldsymbol{\Delta}_{\boldsymbol{\alpha}}^{\mathbf{0}}$ )?

Theorem 3.1 below answers these questions, and determines uniquely the true Borel class of $P(X)$ from the true Borel class of $X$; it says: $P(X)$ is Polish if $X$ is Polish, and for every $\alpha \geq 3, P(X)$ is true $\boldsymbol{\Pi}_{\boldsymbol{\alpha}}^{\mathbf{0}}$ if $X$ is true $\boldsymbol{\Pi}_{\boldsymbol{\alpha}}^{\mathbf{0}}, P(X)$ is true $\boldsymbol{\Pi}_{\boldsymbol{\alpha}+\mathbf{1}}^{\mathbf{0}}$

Received by the editors October 24, 1994 and, in revised form, November 24, 1999.

2000 Mathematics Subject Classification. Primary 03E15, 60B05; Secondary 28A05.

Key words and phrases. Descriptive set theory, probability measures, Borel complexity.

Supported in part by NSF Grant \# DMS-9214048.

(C)2001 Abhijit Dasgupta, GNU GPL style copyleft 
if $X$ is true $\boldsymbol{\Sigma}_{\boldsymbol{\alpha}}^{\mathbf{0}}$, and $P(X)$ is true $\boldsymbol{\Pi}_{\boldsymbol{\alpha}}^{\mathbf{0}}$ if $X$ is true $\boldsymbol{\Delta}_{\boldsymbol{\alpha}}^{\mathbf{0}}$. (These cases are mutually exclusive and exhaustive for Borel $X$.)

In particular, if $X$ is Borel, then either $P(X)$ is Polish or $P(X)$ is true $\boldsymbol{\Pi}_{\boldsymbol{\alpha}}^{\mathbf{0}}$ for some (unique) $\alpha \geq 3$, so that if $\alpha \geq 3$, there is no $X$ such that $P(X)$ is true $\boldsymbol{\Sigma}_{\boldsymbol{\alpha}}^{\mathbf{0}}$ or true $\boldsymbol{\Delta}_{\boldsymbol{\alpha}}^{\mathbf{0}}$.

\section{TERMinology}

We use the notation of [1] and 4 for the Borel pointclasses: the additive, multiplicative, and ambiguous classes of level $\alpha$ are denoted, respectively, by $\boldsymbol{\Sigma}_{\boldsymbol{\alpha}}^{\mathbf{0}}, \boldsymbol{\Pi}_{\boldsymbol{\alpha}}^{\mathbf{0}}$, and $\boldsymbol{\Delta}_{\boldsymbol{\alpha}}^{\mathbf{0}}$, with $\boldsymbol{\Sigma}_{\mathbf{1}}^{\mathbf{0}}$ denoting the pointclass of open sets. $\Gamma$ is a Borel pointclass if $\Gamma$ is one of $\boldsymbol{\Sigma}_{\boldsymbol{\alpha}}^{\mathbf{0}}, \boldsymbol{\Pi}_{\boldsymbol{\alpha}}^{\mathbf{0}}$, and $\boldsymbol{\Delta}_{\boldsymbol{\alpha}}^{\mathbf{0}}$. If $X$ is a Polish space, we use the notation $\boldsymbol{\Sigma}_{\boldsymbol{\alpha}}^{\mathbf{0}} \uparrow X$ to denote the $\boldsymbol{\Sigma}_{\boldsymbol{\alpha}}^{\mathbf{0}}$ subsets of $X$, and similarly for $\boldsymbol{\Pi}_{\boldsymbol{\alpha}}^{\mathbf{0}}$ and $\boldsymbol{\Delta}_{\boldsymbol{\alpha}}^{\mathbf{0}}$. Note that for $\alpha \geq 2$ the pointclass $\boldsymbol{\Pi}_{\boldsymbol{\alpha}}^{\mathbf{0}}$ is intrinsic 1 and for $\alpha \geq 3$ the pointclasses $\boldsymbol{\Sigma}_{\boldsymbol{\alpha}}^{\mathbf{0}}$ and $\boldsymbol{\Delta}_{\boldsymbol{\alpha}}^{\mathbf{0}}$ are also intrinsic. For an intrinsic pointclass $\Gamma$, we can speak (unambiguously) of a separable metrizable space $X$ being $\Gamma$, without mentioning any Polish space in which $X$ is embedded. The notion of "the true Borel class" of a Borel set is defined in the usual way.

$\boldsymbol{\omega}^{\boldsymbol{\omega}}$ denotes the Baire space, i.e., the space of irrationals. If $X, Y$ are Polish spaces, $B \subseteq Y$, and $\mathcal{C}$ is a collection of subsets of $X$, we say that $B$ is $\mathcal{C}$-hard if for all $C \in \mathcal{C}$ there is a continuous $f: X \rightarrow Y$ such that $C=f^{-1}(B)$.

If $X$ is a metrizable space, $P(X)$ denotes the space of probability measure: 2 on $X$ with the weak topology 3

For a separable metrizable space $Y$, and a Borel $X \subseteq Y$, we can (topologically) identify (see [1]) the space $P(X)$ with the subspace $P(X / Y)$ of $P(Y)$, where

$$
P(X / Y) \stackrel{\text { def }}{=}\{\mu \in P(Y) \mid \mu(Y \backslash X)=0\} .
$$

\section{The Borel Complexity of $P(X)$}

Theorem 3.1. If $X$ is any non-Polish Borel subspace of a Polish space, then $P(X)$ is true $\boldsymbol{\Pi}_{\boldsymbol{\xi}}^{\mathbf{0}}$, where $\xi$ is the least such that $X$ is $\boldsymbol{\Pi}_{\boldsymbol{\xi}}^{\mathbf{0}}$.

Proof. We will use the following two lemmas:

Lemma 3.2 (Louveau and Saint Raymond). If $Y$ is a Polish space, $\alpha$ is a countable ordinal $\geq 2, A \subseteq Y$ such that $A$ is Borel, and $A \notin \mathbf{\Pi}_{\boldsymbol{\alpha}}^{\mathbf{0}}$, then $A$ is $\boldsymbol{\Sigma}_{\boldsymbol{\alpha}}^{\mathbf{0}}\left\lceil\boldsymbol{\omega}^{\boldsymbol{\omega}}\right.$-hard, i.e. for all $B \subseteq \boldsymbol{\omega}^{\boldsymbol{\omega}}$, if $B$ is $\boldsymbol{\Sigma}_{\boldsymbol{\alpha}}^{\mathbf{\alpha}}$, then $B=f^{-1}(A)$ for some continuous $f: \boldsymbol{\omega}^{\boldsymbol{\omega}} \rightarrow Y$.

Proof. This is an immediate consequence of [3] Theorem 3, p. 455].

Lemma 3.3 (The $\delta$-propagation lemma). Let $Y, Z$ be Polish spaces, $\mathrm{C}$ a collection of subsets of $Z$, and $X$ a subset of $Y$ which is $\mathcal{C}$-hard, i.e. for every $A$ in $\mathcal{C}$ there is a continuous map $f$ from $Z$ to $Y$ such that $A=f^{-1}(X)$. Then $P(X / Y)$ is $\mathcal{C}_{\delta}$-hard, where $\mathcal{C}_{\delta}$ denotes the countable intersections of sets in $\mathcal{C}$.

\footnotetext{
${ }^{1} \Gamma$ is an intrinsic pointclass if for all Polish $X, Y$ and $A \subseteq X, B \subseteq Y$, if $A$ is a $\Gamma$-set in $X$ and $A$ is homeomorphic to $B$, then $B$ is a $\Gamma$-set in $Y$.

${ }^{2}$ The collection of all countably additive non-negative real-valued functions $\mu$ defined on the Borel sets of $X$ such that $\mu(X)=1$.

${ }^{3}$ The weakest topology such that, for every bounded continuous real-valued function $f$ on $X$, the real-valued map $\mu \rightarrow \int f d \mu$ defined on $P(X)$ is continuous.
} 
Proof. Let $X, Y, Z$, and $\mathcal{C}$ be as above. To show that $P(X / Y)$ is $\mathcal{C}_{\delta}$-hard, let $F$ be an arbitrary subset of $Z$ in $\mathcal{C}_{\delta}$. Then there is sequence $\left(E_{n}\right)_{n=0}^{\infty}$ of subsets of $Z$ such that $(\forall n)\left(E_{n} \in \mathcal{C}\right)$, and

$$
F=\bigcap_{n=0}^{\infty} E_{n}
$$

For each $n \in \omega$, choose a continuous function $f_{n}: Z \rightarrow Y$ such that

$$
E_{n}=f_{n}^{-1}(X)
$$

Define $\Psi: Z \rightarrow P(Y)$ by setting for each $x \in Z$ and each Borel $E \subseteq Y$ :

$$
\Psi(x)(E) \stackrel{\text { def }}{=} \sum_{k=0}^{\infty} \frac{\chi_{E}\left(f_{k}(x)\right)}{2^{k+1}},
$$

where, for any set $A, \chi_{A}$ denotes the characteristic function of $A$. In other words, for any $x \in Z, \Psi(x)$ is the measure

$$
\Psi(x)=\sum_{k=0}^{\infty} \frac{1}{2^{k+1}} \delta_{f_{k}(x)},
$$

where, for any $y \in Y, \delta_{y}$ denotes the probability measure on $Y$ known as the "unit mass" at $y . \quad \Psi$ is well-defined and continuous. It is now easy to verify that $\Psi$ reduces $F$ to $P(X / Y)$.

Now let $X$ be non-Polish Borel, and $Y$ be any metrizable compactification of $X$; let $\alpha$ be the least such that $X$ is $\boldsymbol{\Pi}_{\boldsymbol{\alpha}}^{\mathbf{0}}$. Then $\alpha \geq 3$, as $X$ is not $\boldsymbol{\Pi}_{\mathbf{2}}^{\mathbf{0}}$ in $Y$. Also, $P(X)$ is $\boldsymbol{\Pi}_{\boldsymbol{\alpha}}^{\mathbf{0}}$. It remains to show that $P(X)$ is not $\boldsymbol{\Sigma}_{\boldsymbol{\alpha}}^{\mathbf{0}}\left(\boldsymbol{\Sigma}_{\boldsymbol{\alpha}}^{\mathbf{0}}\right.$ is an intrinsic pointclass since $\alpha \geq 3)$. Put $Z=\omega^{\omega}$, and

$$
\mathcal{C}=\bigcup_{\beta<\alpha} \Sigma_{\beta}^{0}\left\lceil\omega^{\omega} .\right.
$$

By Lemma $3.2 X$ is $\boldsymbol{\Sigma}_{\boldsymbol{\beta}}^{\mathbf{0}}\left\lceil\boldsymbol{\omega}^{\boldsymbol{\omega}}\right.$-hard for every $\beta<\alpha$, and hence is $\mathcal{C}$-hard. The hypotheses of the $\delta$-propagation lemma now hold. Therefore $P(X / Y)$ is $\mathcal{C}_{\delta}$-hard; but $\mathcal{C}_{\delta}=\boldsymbol{\Pi}_{\boldsymbol{\alpha}}^{\mathbf{0}}\left\lceil\boldsymbol{\omega}^{\boldsymbol{\omega}}\right.$. So $P(X)$ must be true $\boldsymbol{\Pi}_{\boldsymbol{\alpha}}^{\mathbf{0}}$.

It follows from the above theorem that the Borel complexity of the space of probability measures on $\mathbb{Q}$ (the rationals) is $\mathcal{F}_{\sigma \delta}$ but not $\mathcal{G}_{\delta \sigma}$.

\section{REFERENCES}

[1] A. S. Kechris, Classical Descriptive Set Theory, Springer-Verlag, 1995. MR 96e:03057

[2] A. S. Kechris, Measure and category in effective descriptive set theory, Annals of Math. Logic 5 (1973), 337-384. MR 51:5308

[3] A. Louveau and J. Saint Raymond, Borel classes and closed games: Wadge-type and Hurewicztype results, Trans. Amer. Math. Soc. 304 (1987), 431-467. MR 89g:03068

[4] Y. N. Moschovakis, Descriptive Set Theory, North-Holland, 1980. MR 82e:03002

[5] K. R. Parthasarathy, Probability Measures on Metric Spaces, Academic Press, 1967. MR 37:2271

[6] S. Shreve, Probability measures and the C-sets of Selivanovskij, Pacific J. Math. 79 (1978), 189-196. MR 80d:28008

E-mail address: takdoom@yahoo.com 